\title{
Design and Realization of Improved Image Restoration Method Based on Fuzzy Projection onto Convex Sets
}

\author{
Xin Li \\ Guangzhou College of South China University of Technology, Guangzhou, 510800, China \\ lixin-820701@163.com
}

\begin{abstract}
Noise affects the recovery effect. So this paper proposed an improved method for image restoration. In this method, first of degraded images using wavelet transform to remove noise while fuzzy POCS algorithm to measure the reliability of the wavelet coefficients, so while eliminating noise can incorporate more reliable information. Experimental results show that the proposed approach in image restoration can effectively eliminate noise and improve image clarity.
\end{abstract}

Keywords-wavelet transform; fuzzy POCS; image restoration; reliability

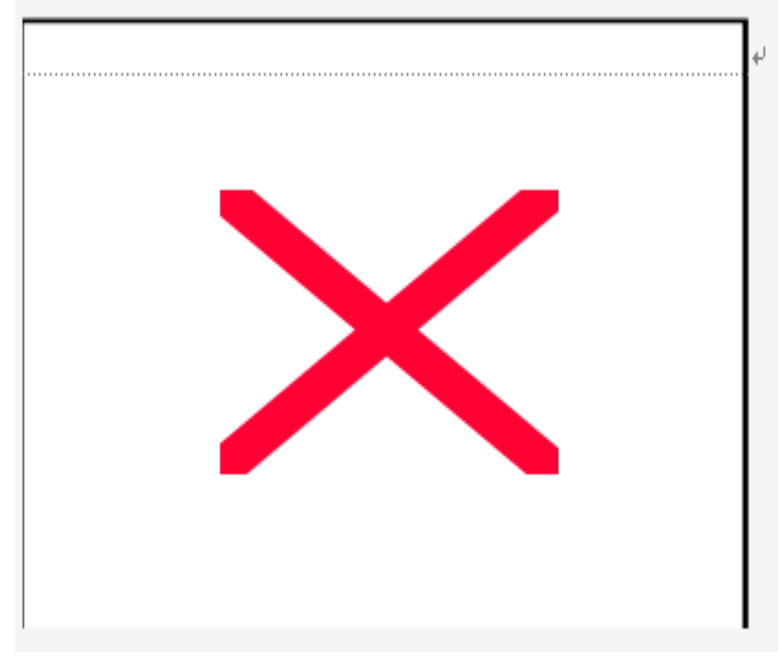

\section{INTRODUCTION}

Image restoration, image deblurring technology in particular, has been a hot topic in image processing. In recent years, there are many domestic and foreign scholars have conducted research, made a number of sophisticated image restoration algorithms, such as conventional POCS (POCS) ${ }^{[1-3]}$ algorithm, Lucy-Richardson (LR) ${ }^{[4-5]}$ algorithm, the literature [6] algorithm, but also can not effectively solve the problem of image restoration morbid. On the one hand, a lot of image restoration methods are based on the known point spread function (PSF) for image restoration, such as conventional POCS algorithm. Indeed, PSF is often unknown, it is necessary to estimate the frequency domain or spatial domain, but the estimated PSF are difficult to ensure its accuracy. On the other hand, The existence of noise in the image restoration of blurred and degenerated images containing noise will influence result of image restoration, especially in low SNR conditions. So noise must be effectively filtered out in image restoration process.

Combining the denoising and deblurring need, the article presents the way of image restoration with Wavelet Transform $^{[7-8]}$ and fuzzy POCS. In terms of denoising, the introduction of the wavelet transform, which has the characteristic of multi-resolution analysis and wavelet packet analysis features and the function of characterization of local signal characteristics in two space-time domain, is very suited for noisy signal detection analysis and showing its components .For deblurring, we use fuzzy POCS for wavelet decomposition of the different observing images of the same scene to identify the wavelet coefficients of reliability .This will not only eliminate the influence of noise, and can ensure that the results of image fusion and reliable information as much as possible.

\section{PoCs FuZZY DEFINITION}

Fuzzy POCS algorithm of this paper ,similar to conventional POCS algorithm, through integration of multiple observations (degraded) image information to achieve lower image noise and improve image clarity, only runs in the wavelet domain. According to the linear system theory ${ }^{[9]}$, the observed image degradation model multi-system can be expressed as follows:

$$
g_{i}=h_{i} * f+n_{i} \quad i=1,2, \ldots . N
$$

$f$ represents an original image and $g_{i}, h_{i}, n_{i}$ are for the i-th observation (degraded) image, the i-th point spread function of the observation image (PSF), and the i-th images of the additive noise (typically Gaussian noise) respectively.

While the noise and blur of observation image and the presence of fuzzy wavelet coefficients observation image degradation and distortion exist, the information contained in the different observing different images, so it is possible to restore or reduce distortion.

In order to measure the degree of distortion of the wavelet coefficients, this paper introduces a fuzzy projection onto convex sets, which is defined as follows:

$$
A_{f}^{\alpha}=\left\{\begin{array}{lr}
\left\{x \mid \mu_{A}(x) \geq \alpha, x \in E\right\}, & \alpha \neq 0 \\
E, & \text { others }
\end{array}\right.
$$

The formula means that the definition of fuzzy sets in E, $A_{f}^{\alpha}$ is the $\alpha$ truncation of $A_{f} \quad(0 \leq \alpha \leq 1)$, and for the 
membership function $\mathrm{E}$ on the range $[0,1]$, indicating the degree of E's membership. Common set of $\mathrm{A}, \mu_{A}(x)$ is defined as:

$$
\mu_{A}(x)=\left\{\begin{array}{l}
1, x \in A \\
0, \text { others }
\end{array}\right.
$$

In order to meet the definition of convex sets, only:

$$
\mu_{\mathrm{A}}[\lambda \mathrm{a}+(1-\lambda) \mathrm{b}] \geq \min \left\{\mu_{A}(\mathrm{a}), \mu_{A}(\mathrm{~b})\right\}
$$

Where $0 \leq \lambda \leq 1$

$$
\mu_{\text {A }}\left(\mathrm{G} \quad{ }_{i}^{l}(u, v)\right)=
$$

$\gamma_{l}(l=1,2, \ldots, \mathrm{L})$ is positive, taking into account the more advanced wavelet detail coefficients by the greater role of the low-pass filter, the less affected by noise, so in the case of the same magnitude, and its membership should be attached to the high than the low-level details of the wavelet coefficients, like $\gamma_{1} \leq \gamma_{2} \leq \ldots \leq \gamma_{L}$.Meanwhile, the wavelet transform coefficients of the approximation should be reliable, so the membership should be 1 . To facilitate

\section{ALGORITHM}

\section{A. Algorithm principle}

As the absolute value of wavelet coefficients for image are larger, the noisy and blur effect seldom influence the image and the image is more credible, so the wavelet coefficients are more possible to closer to wavelet coefficients of the original image. Therefore, let each piece of observation images $g_{i}(i=1,2, \ldots, N)$ of $\mathrm{L}$ level wavelet decomposition, $\mathrm{G}_{i}^{l}(u, v)$ of the level $\mathrm{L}$ of detail coefficients of wavelet, its membership can be defined as:

- $\mathrm{e} x \mathrm{p}\left(-\gamma, \mathrm{G}{ }_{i}^{l}(u, v)\right)$

consistent, the image $g_{i}(i=1,2, \ldots, N)$ is $\mathrm{L}$ level wavelet decomposition approximation and detail coefficients at all levels of the sub-picture coefficient referred to as the combined image and set toward $G_{i}(i=1,2, \ldots, \mathrm{N})$, the membership function can be expressed as unity:

$$
\mu_{\mathrm{A}}\left(G_{i}(\mu, v)\right)= \begin{cases}1-\exp \left(-\gamma_{1} G\right), G_{i}(\mu, v) & \text { is level }-\mathrm{L} \text { detail coefficien } \\ 1, G_{i}(\mu, v) \text { is approximat } & \text { e coefficien ts }\end{cases}
$$

From the definition of membership function of (6) to see a collection of his correspondence is a convex set, define the wavelet coefficients to the projection of convex sets as follows:

$$
p^{\alpha}\left(\mathrm{G}_{i}^{l}(u, v)\right)= \begin{cases}0, & \mu_{\mathrm{A}}\left(\mathrm{G}_{i}^{l}(u, v)\right)<\alpha \\ \mathrm{G}_{i}^{l}(u, v), & \text { other s }\end{cases}
$$

Wavelet coefficients projected onto convex sets significance lies in the credibility of the wavelet coefficients dropped too low (set to 0 ), to ensure that the information (wavelet coefficients) the credibility of the algorithm results.

Further wavelet coefficient defined from expression of the greater degree of membership which are subjected to degradation factors, the higher the degree of confidence concept, in order to ensure the final image fusion have as much reliable information, in the wavelet domain for each observation ( $\mathrm{L}$ level wavelet coefficients image) $\mathrm{G}_{i}$ define the image of a convex set, and (L-level image wavelet coefficients) of the original image to the projection $\mathrm{F}$ convex sets:

$$
C_{i}=\left\{F(u, v) \mid a b s(F(u, v)) \geq \operatorname{abs}\left(G_{i}(u, v)\right)\right\}
$$

Projection for:

$P_{F}^{i} F(u, v)= \begin{cases}F(u, v), a b s(F(u, v)) \geq a b s\left(G_{i}(u, v)\right) \\ G_{i}(u, v), & \text { others }\end{cases}$

Because of the above definition of a uniform set of membership functions and convex projection, for convenience, the following statements are no longer distinguish approximate coefficients and detail coefficients at all levels.

\section{B. Algorithm steps}

With the above definition and analysis, fuzzy POCS algorithm proposed process can be expressed as follows:

Step 1: Initialize $\alpha_{\max }, \alpha_{\min }, \alpha_{\text {stp }}$ and $\gamma_{l}(l=1,2, \ldots, \mathrm{L})$, let each observation image $g_{i}(i=1,2, \ldots, N)$ do level $L$ wavelet decomposition for the image $G_{i}(i=1,2, \ldots, N)$ of wavelet coefficients ;

Step 2: Let $\alpha=\alpha_{\text {max }}, i=1, \hat{F}=G_{l}, \hat{F}$ image shows the approximative image of the original image of the level $\mathrm{L}$ wavelet coefficients ;

Step 3: For each wavelet coefficients $\hat{F}(u, v)$ all of them projected onto convex

sets $\mathrm{C}_{i}: \hat{F}(u, v)=\mathrm{P}_{\hat{F}}^{i}(\hat{F}(u, v))$;

Step 4: If $i=N$, you go to step 5 , otherwise $i=i+1$, return to step 3;

Step 5: For each wavelet coefficients $\hat{F}(u, v)$ projected onto convex sets $A_{f}^{\alpha}$ can be expressed as $\hat{F}(u, v)=\mathrm{P}^{\alpha}(\hat{F}(u, v))$;

Step 6:, if $\alpha=\alpha-\alpha_{\text {step }}$, and the next step is step 7. 
Otherwise, it go to step 3;

Step 7: The final fused image $\hat{f}$ of the inverse wavelet transform for $\hat{F}$ is an estimate of the original image f.

The above steps, Step 3 ensures that the final image as a fusion with a maximum of the most important information, steps 5 to ensure the reliability of the integration of information, and through the loop of Step 6, is reduced gradually so that the convex set is gradually expanded, thereby so that each of the wavelet coefficients based on the reliability of their order to be included in the phase.

\section{COMPARATIVE EXPERIMENT}

Experiments are carried out on Matlab platform. Experimental use "sym4" wavelet for experiments of five class wavelet decomposition, setting

$$
\alpha_{\max }=0.99, \alpha_{\text {min }}=0.05, \alpha_{\text {step }}=0.01 \gamma_{1}=5.0 e-3,
$$

$\gamma_{2}=\gamma_{3}=4.5 e-3, \gamma_{4}=\gamma_{5}=4.0 e-3$, and produced five degraded images. The production of degraded images is based on the image degradation model (check expression 1) , and each of PSF from the degraded image is generated by motion blur and Gaussian Blur convolution ${ }^{[10]}$, and $\sigma_{n}^{2}=5$ of Gaussian White Noise is set for additive noise .

In the experiments, the algorithm on the paper was compared with Lucy-Richardson (LR) algorithm and reference [6] algorithm. Figure 1 is the results of experiment for the Lena image of $512 \times 512$ size. The figure 1 (a) of them is the one of degraded images which are added by blur and noise .

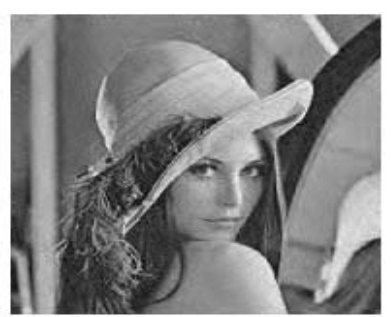

a

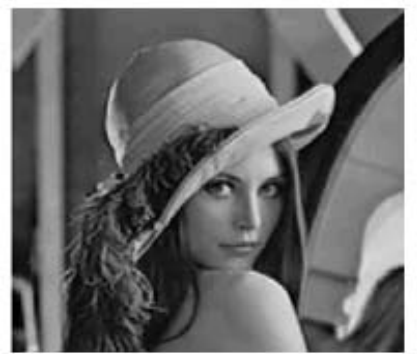

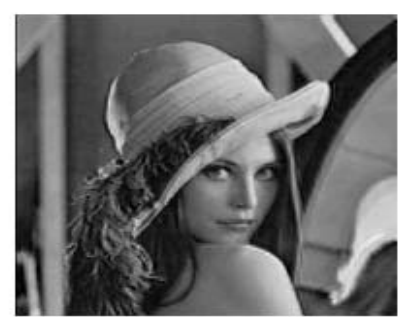

b

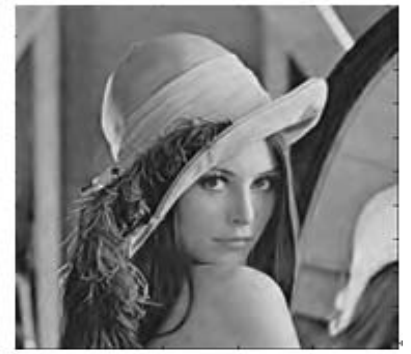

$\mathrm{d}$ a. Lena Degradation image, b. The result of Lucy-Richardson algorithm restoration, c. The result of recovery of the method of the reference[6], d. The results of the recovery of algorithm on the paper

Figure. 1 Lena restoration effect comparison chart

In order to objectively evaluate the results of recovery of each algorithm, I introduce PSNR, running time of the algorithm , and the relevant measure $\mathrm{K}$ for image restoration,K expression as follow:

$$
K=\frac{\sum_{i=0}^{M-1} \sum_{j=0}^{N-1} f(i, j) g(i, j)}{\left[\sum_{i=0}^{M-1} \sum_{j=0}^{N-1} f^{2}(i, j) \sum_{i=0}^{M-1} \sum_{j=0}^{N-1} g^{2}(i, j)\right]^{\frac{1}{2}}}
$$

Their comparison results are shown below in Table 1:

\begin{tabular}{|c|c|c|c|}
\hline \multirow[t]{2}{*}{ Image } & \multicolumn{3}{|c|}{ PSNR algorithm（dB） } \\
\hline & L-R algorithm & [6] algorithm & Algorithm \\
\hline \multirow{7}{*}{ Lena } & 24.98 & 29.67 & 35.01 \\
\hline & \multicolumn{3}{|c|}{ Algorithm running time (Second) } \\
\hline & L-R algorithm & [6] algorithm & Algorithm \\
\hline & 5.604 & 1.018 & 1.481 \\
\hline & \multicolumn{3}{|c|}{$\mathrm{K}$} \\
\hline & L-R algorithm & [6] algorithm & Algorithm \\
\hline & 1.1207 & 1.1109 & 1.045 \\
\hline
\end{tabular}

TABLE I. THE RESULTS OF PSNR OF RESTORATION IMAGE OF EACH ALGORITHM

As can be seen from Table I, the proposed algorithm achieved ratio LR algorithm [6] better restoration effect, because LR algorithm using only the information in a degraded image restoration, while the literature [6] and use the algorithm for five degraded image information fusion method, the original image information thus acquired more and better recovery results; in addition, the literature [6] in the frequency domain using the recovery method, according to the reliability coefficient, rounding unreliable factor (set to 0 ), and therefore its great performance and fuzzy performance denoising conflict, and algorithms used in the wavelet domain image restoration using a wavelet transform features, enabling different levels of detail in the image with varying degrees of processing, reducing the impact on the denoised image deblurring. From the correlation measure $K$ values can also be seen that the algorithm is closer to 1 , which is the three algorithms, the proposed algorithm for image restoration error is minimized, is closer to the original image on the visual senses. Considering the above three indicators, the best algorithm 
for image restoration.

In order to investigate the observed image (degraded image) affects the number of images on the fusion results, the following number of Lena test image in the case of 3,5,8 and 10, as showed in Table 2:

TABLE II. THE EFFECT ON THE OBSERVED NUMBER OF IMAGES FUSED IMAGE

\begin{tabular}{c|c|c}
\hline Lena & The number of images & 3 \\
\hline & PSNR $(\mathrm{dB})$ & 32.02 \\
\hline
\end{tabular}

Note from Table II, the number of images within a certain range, the observed image (degraded image) obtained the number, the higher the quality of the restored image, the closer the original image. However, when the number of observations increases to five or more images, image restoration quality and not on the number of images increases. This is because the number of the observed image to a certain extent, the newly added observation image cannot bring more new information, leading to the restored image PSNR slow growth or essentially unchanged.

\section{V.CONCLUSIONS}

This paper presents image restoration algorithm based on wavelet transform and fuzzy POCS combine. The algorithm needn't using PSF in dealing with image blur through fusing the image information of wavelet decomposition to improve the clarity of final images. The introduction of POCS fuzzy wavelet coefficients in the image fusion process ensure the reliability of the fusion process, but also effectively suppresses noise. Finally, the fusion in wavelet domain can take advantage of the characteristics of wavelet transform to reduce the impact of noise on the image fusion effect, saving the running time of the algorithm to improve the image restoration effect.

\section{REFERENCES}

[1]Tian Xie. POCS algorithm using an improved super-resolution image

\begin{tabular}{c|c|c}
5 & 8 & 10 \\
\hline 34.92 & 34.97 & 34.98 \\
\hline
\end{tabular}

restoration [J] Electronic Design Engineering, 2013, 21 (18): 142-143. In Chinese.

[2] Sabati Mohammad,Peng Haidong,Lauzon M Louis,Frayne Richard. A statistical method for characterizing the noise in nonlinearly reconstructed images from undersampled MR data: The POCS example [J]. Magnetic resonance imaging, 2013, 31 (9):1587-1598.

[3] Jianjun Gao, Aaron Stanton, Mostafa Naghizadeh, Mauricio D. Sacchi, Xiaohong Chen. Convergence improvement and noise attenuation considerations for beyond alias projection onto convex sets reconstruction[J]. Geophysical Prospecting, 2013, 61:138-139.

[4] He Yan, Weijun Yan, Weiwei Li. restored image Lucy-Richardson algorithm [J]. Computer Engineering, 2010.36 (15): 204-205. In Chinese.

[5] Hojjatoleslami S A,Avanaki M R N,Podoleanu A Gh. Image quality improvement in optical coherence tomography using Lucy-Richardson deconvolution algorithm[J]. Applied optics, 2013, 52 (23):5663-5670.

[6] Seung-Won Jung, Tae-Hyun Kim, and Sung-Jea Ko. A Novel Multiple Image Deblurring Technique Using Fuzzy Projection onto Convex Sets[J]. IEEE Signal Processing Letters, 2009, 3(16):245-249.

[7] Haidong LI, Qing LI. algorithm de-noising based on wavelet thresholding [J].Computer Technology and Development, 2009, 19 (7): 56-58. In Chinese.

[8] K. Kannan,S. Arumuga Perumal,K. Arulmozhi. Performance Comparison of various levels of Fusion of Multi-focused Images using Wavelet Transform[J]. International Journal of Computer Applications, 2010, 1 (6):71-72.

[9] YuJin Zhang. Image Processing (3rd Edition) (Image Engineering, the book) [M]. Beijing: Tsinghua University Press, 2012: 101-103. In Chinese.

[10] S. Battiato, G. Gallo, F. Stanco. A new edge-adaptive algorithm for zooming of digital images [J]. In Proceedings of IASTED Signal Processing and Communications SPC, 2000, 16(10): 144-149. 